\title{
Correction of a nasal soft triangle deficiency as a complication of augmentation rhinoplasty
}

\author{
Eon Su Kim, \\ Jae Won Heo, \\ Chae Eun Yang, \\ Jiye Kim, \\ Sug Won Kim \\ Department of Plastic and \\ Reconstructive Surgery, Yonsei \\ University Wonju College of Medicine, \\ Wonju, Korea
}

\begin{abstract}
The soft tissue triangle is an easily recognizable subunit of the nose. Therefore, deformities in this region resulting from trauma or complications after cosmetic surgery can have serious cosmetic impacts. Various reconstruction choices exist for deformities such as depression of the soft triangle but choosing the most appropriate treatment in each case remains a challenge. In the case described herein, a patient underwent augmentation rhinoplasty with a silastic implant and experienced implant exposure in the soft triangle area. After implant removal, the patient complained of depression in this area. The authors effectively solved this problem through a de-epithelialized composite tissue graft. In this report, we present this case and review similar cases of reconstruction of the soft triangle.
\end{abstract}

Keywords: Cosmetic reconstructive surgery / Nose deformities, acquired / Tissue transplantation

\section{INTRODUCTION}

Since the nose is a structure located in the middle of the face, loss or deformation of the nose has a significant cosmetic impact. The soft triangle, which is the area between the dome and the nostril rim, is a critical component of the aesthetic aspect of the nose (Fig. 1) [1,2]. Deformities of the soft triangle, which can occur due to trauma or postoperative infection, pose considerable difficulties for successful cosmetic reconstruction. Various methods, such as composite grafts, paramedian forehead flaps, and nasolabial flaps, have been attempted, but choosing the most appropriate treatment in each case remains a challenge due to the need to consider factors such as color, texture, and flexibility $[3,4]$. In this report, we present a case where depressive scar contracture of the soft triangle and nostril asymmetry were effectively corrected through a composite graft.

\section{Correspondence: Sug Won Kim}

Department of Plastic and Reconstructive Surgery, Yonsei University Wonju

College of Medicine, 20 Ilsan-ro, Wonju 26426, Korea

E-mail: sugwonkim@yonsei.ac.kr

Received April 10, 2021 / Revised May 27, 2021 / Accepted June 10, 2021

\section{CASE REPORT}

A 50-year-old woman, who had undergone augmentation rhinoplasty using a silastic implant in a local aesthetic clinic 6 years previously, had experienced implant exposure due to postoperative infection. Removal of the exposed implant 3 months after the operation had resulted in scar contracture of the soft triangle site with depression. The patient also complained of nostril asymmetry resulting from the soft triangle depression (Fig. 2). Surgery was performed under general anesthesia. After full excision of the depressed scar, the defect volume was measured using soaked gauze. Composite tissue containing skin and soft tissue was harvested in the preauricular area. After partial de-epithelialization (Fig. 3) the harvested tissue was fixed using key sutures with nylon \#6-0 (Fig. 4). Immediately after the operation, local hyperbaric therapy was applied on the composite graft site for 7 days.

The grafted tissue was well taken, without necrosis. The stitches were removed on postoperative day 7 . At an outpatient follow-up visit 3 weeks later, the soft triangle depression was corrected, and the asymmetry of the nostril seen before the op- 


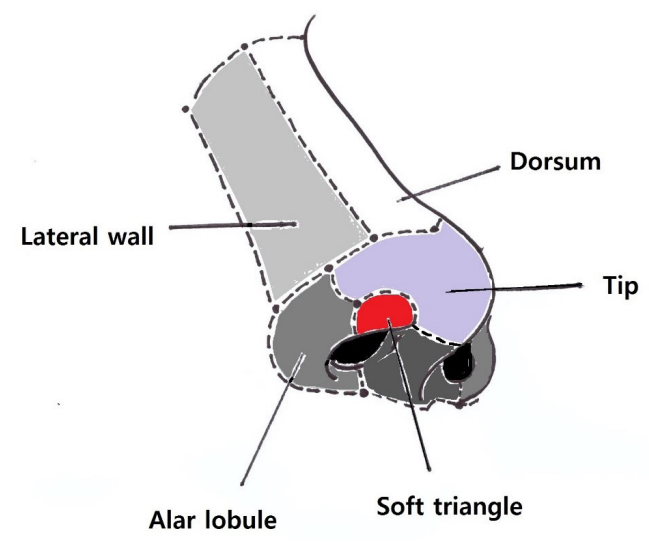

Fig. 1. Aesthetic subunits. The soft triangle is the area between the dome and the nostril rim.
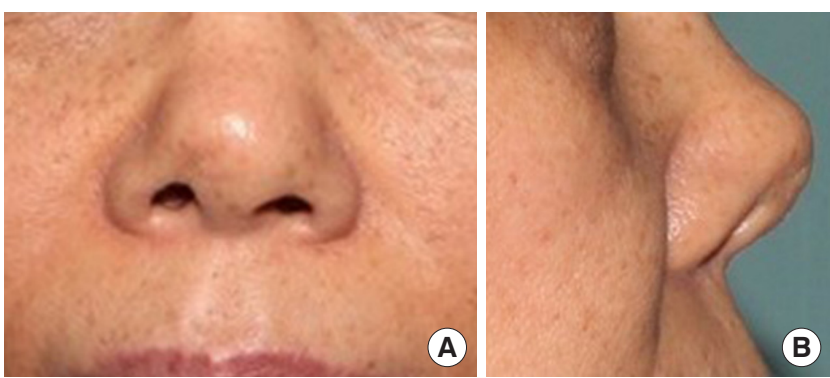

Fig. 2. A 50-year-old woman. The appearance of the nose shows postoperative scar contracture. (A) Frontal view and (B) lateral view.

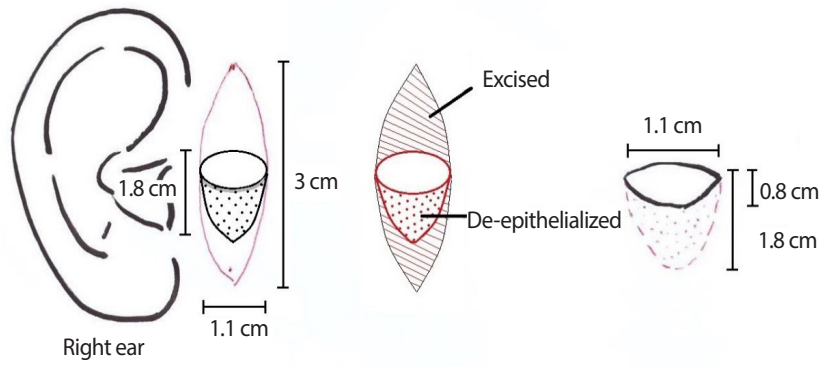

Fig. 3. Schematic image of the design of the composite tissue. The size of the tissue needed for the graft is $1.1 \times 1.8 \mathrm{~cm}$ with a $1.1 \times 0.8 \mathrm{~cm}$ skin paddle. For primary closure of the donor site, elliptical tissue measuring $3 \times 1.1 \mathrm{~cm}$ was harvested on the ipsilateral side.

eration due to contracture had also improved (Fig. 5).

\section{DISCUSSION}

Deformity of the soft triangle is likely to occur after trauma or infection, and even a small deformity has a major effect on the shape of the nose and the overall aesthetic outcome $[5,6]$. Of all nine subunits of the nose, the soft triangle is perhaps the most challenging to reconstruct [4]. The soft triangle is a specific anatomic subunit of the nose, located between the dome and alar
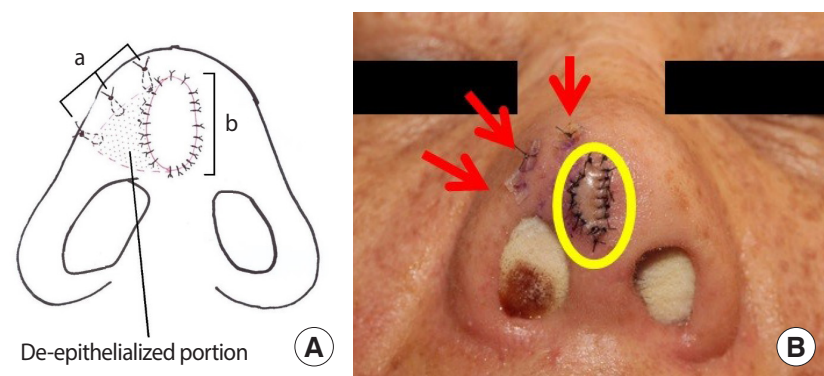

Fig. 4. (A) Schematic image of the postoperative state. De-epithelialized tissue was placed under the contracture area to resolve the soft tissue depression. Key sutures were used to fix the de-epithelialized portion (a), the skin paddle was sutured with skin sutures (b). (B) Immediate postoperative appearance of the nose. Key suture (stitch a, red arrows) and composite graft (stitch b, yellow circle).
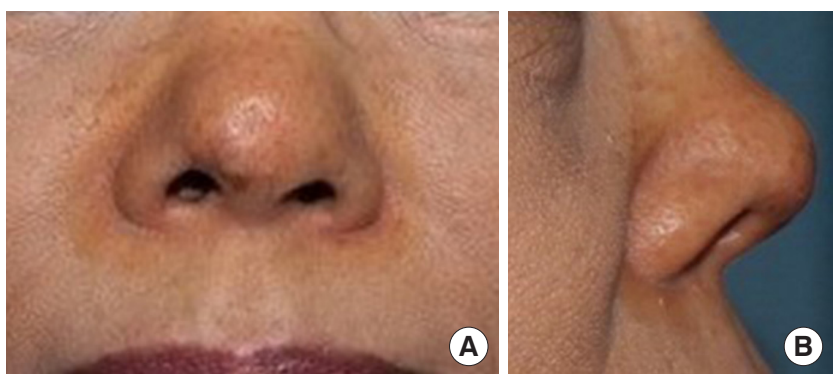

Fig. 5. Postoperative photographs 3 weeks after the composite graft. The depression and asymmetry of the nostril had improved. (A) Frontal view and (B) lateral view.

lobule, and it presents an aesthetic subunit, as proposed by Burget and Mernick (Fig. 1) [1]. According to previous research, this area is "the only place in humans where skin abuts skin directly, without any intervening soft tissue" [7].

A recent detailed study of the soft triangle demonstrated that this region can be divided into three zones according to histological characteristics. Zone 1 is where the fibers of the dilator naris anterior muscle insert into the dermis of the skin tissue located between the lateral and medial crus. In zone 2, the areolar tissue is sandwiched between the dermis of the external and internal layers. Finally, zone 3 is the site where the fibers of the nasalis muscle and depressor septi muscle interdigitate with the dermis [8]. Considering both aesthetic concerns and nasal function, surgical incision or trauma should be avoided in this area.

Reconstruction of the soft triangle should be performed with due consideration of these anatomical features, and thus requires an in-depth understanding of the relevant issues. In previous studies, full-thickness skin grafts were used to reconstruct small defects. For the reconstruction of larger defects, a turnover flap from the inferior tip covered with a full-thickness skin graft has been used [9], and other reconstructive options in- 
clude local flaps, such as nasolabial, trilobed, or nasalis sling island flaps $[4,10]$. In the case presented herein, depression and nostril asymmetry were the main targets of correction due to scar contracture through the soft triangle from the inside. Therefore, this scar tissue was completely excised and released. Thicker tissue was then needed to reinforce the depression rather than thin tissue such as that used in a full-thickness skin graft. Therefore, in the composite graft, the tissue included a skin paddle to cover the excised skin area of the surface and a de-epithelialized portion to reinforce the depression. The deepithelialized portion was fixed with a key suture-shown as "stitch a" in the figures-after undermining the depressed site. The skin paddle was fixed with a general suture (Fig. 4). Through this modified composite graft, we seem to have successfully solved the problem posed by the depression of the soft triangle in this case.

As mentioned above, the soft triangle is a unique area of the human body, which is crucial to understand thoroughly for reconstructive procedures. It is particularly necessary to consider this area from a 3-dimensional standpoint because of its involvement with the alar lobules, domes, and nostrils. Our results demonstrate that a partially de-epithelialized composite graft is a preferred choice for volume deficiency due to scar contracture after removal of the implant, and reconstruction can be performed with minimal donor site morbidity.

\section{NOTES}

\section{Conflict of interest}

No potential conflict of interest relevant to this article was reported.

\section{Ethical approval}

The study was approved by the Institutional Review Board of Wonju Severance Christian Hospital (IRB No. CR321314) and performed in accordance with the principles of the Declaration of Helsinki. Written informed consent was obtained.

\section{Patient consent}

The patient provided written informed consent for the publication and the use of her images.

\section{ORCID}

Eon Su Kim

https://orcid.org/0000-0001-5938-9645

Jae Won Heo https://orcid.org/0000-0002-9070-5244

Chae Eun Yang

Jiye Kim https://orcid.org/0000-0001-8128-791X https://orcid.org/0000-0002-1724-4250

Sug Won Kim

\section{Author contribution}

Conceptualization: ESK, SWK. Data curation: ESK, JWH. Visualization: ESK, JWH, CEY, JK. Writing - original draft: ESK. Writing - review \& editing: ESK, SWK, CEY, JK.

\section{REFERENCES}

1. Burget GC, Menick FJ. The subunit principle in nasal reconstruction. Plast Reconstr Surg 1985;76:239-47.

2. Kim TK, Jeong JY. Surgical anatomy for Asian rhinoplasty. Arch Craniofac Surg 2019;20:147-57.

3. Choi MH, He WJ, Son KM, Choi WY, Cheon JS. The efficacy of dermofat grafts from the groin for correction of acquired facial deformities. Arch Craniofac Surg 2020;21:92-8.

4. Constantine FC, Lee MR, Sinno S, Thornton JF. Reconstruction of the nasal soft triangle subunit. Plast Reconstr Surg 2013; 131:1045-50.

5. Bayram AA, Kilavuz AE, Serin GM. The importance of soft triangle in rhinoplasty. J Craniofac Surg 2016;27:e536-7.

6. Kayabasoglu G, Nacar A. The soft triangle: an often neglected area in rhinoplasty. Aesthetic Plast Surg 2015;39:659-66.

7. Natvig P, Sether LA, Dingman RO. Skin abuts skin at the alar margins of the nose. Ann Plast Surg 1979;2:428-9.

8. Ali-Salaam P, Kashgarian M, Persing J. The soft triangle revisited. Plast Reconstr Surg 2002;110:14-6.

9. Zitelli JA. Repair of the soft triangle of the nose. J Dermatol Surg Oncol 1994;20:839-41.

10. Goldman GD. Reconstruction of the nasal infratip, columella, and soft triangle. Dermatol Surg 2014;40 Suppl 9:S53-61. 